
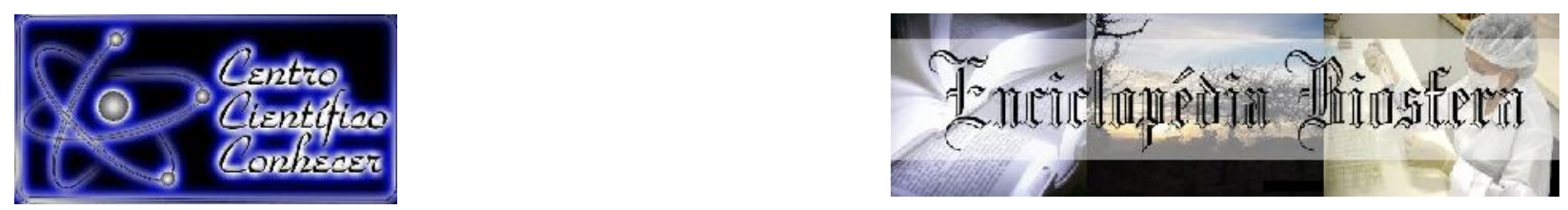

\title{
ESTUDO DA ATIVIDADE ANTIOXIDANTE DO EXTRATO BRUTO HIDROALCOÓLICO DO CAPIM-CIDREIRA (Cymbopogon citratus) PELO MÉTODO DPPH
}

Lanussy Porfiro de Oliveira ${ }^{1}$, Juliene Ribeiro Vieira Barcelos ${ }^{2}$, Sara llana Ferreira de Oliveira $^{2}$, Oscar Romero Lopes Rodrigues ${ }^{1,2}$ e Thiago Sardinha de Oliveira ${ }^{3}$

${ }^{1}$ Faculdade de Farmácia, Universidade Federal de Goiás, Goiânia-GO, Brasil.

${ }^{2}$ Faculdade de Farmácia, Universidade Estadual de Goiás, Itumbiara-GO, Brasil.

${ }^{3}$ IBiotec, Universidade Federal de Goiás, Catalão-GO, Brasil e-mail: thiago_tso@ufg.br

Recebido em: 06/04/2019 - Aprovado em: 10/06/2019 - Publicado em: 30/06/2019 DOI: 10.18677/EnciBio_2019A157

\begin{abstract}
RESUMO
A procura de substâncias alternativas para inibição de radicais livres é crescente, sendo as plantas medicinais uma fonte promissora de compostos com atividade antioxidantes. O capim-cidreira (Cymbopogon citratus) é uma planta amplamente distribuída e utilizada na medicina popular, pertence à família Poaceae e é conhecida por diversos nomes populares, tais como capim-limão, erva-cidreira, capim-santo, etc. Dentre as finalidades terapêuticas no seu uso popular, destacamse as atividades do tipo calmante, analgésica, antiespasmódica, antibacteriana e anticonvulsivante, que podem estar correlacionadas à atividade antioxidante de compostos presentes nesta planta, assim a investigação do potencial antioxidante do capim-cidreira através do método "in vitro" do 2,2 difenil-1-picril hidrazil radical (DPPH) foi o objeto de estudo do presente trabalho. A atividade sequestradora de radicais livres do $\mathrm{EBH}$ das folhas de capim-cidreira mostrou-se significante, demonstrando efeito dependente de concentração, que no caso de plantas aromáticas é comumente atribuída à presença de flavonóides, catequinas e outros compostos fenólicos.
\end{abstract}

PALAVRAS-CHAVE: Capim-cidreira, Cymbopogon citratus, Atividade Antioxidante, Plantas Medicinais e DPPH.

\section{STUDY OF ANTIOXIDANT ACTIVITY OF CAPIM-CIDREIRA HYDROALCOOLIC EXTRACT (Cymbopogon citratus) TROUGH DPPH METHOD}

\begin{abstract}
The search of alternative substances for inhibition of free radicals is increasing, which medicinal plants being a promising source of compounds with antioxidant activity. Lemongrass (Cymbopogon citratus) is a widely distributed plant used in folk medicine, belongs to Poaceae family and known as many popular names, such lemongrass, lemon balm, holy grass, etc. Among therapeutic purposes in popular use are the activities of calming, analgesic, antispasmodic, antibacterial and anticonvulsive, which can be correlated to antioxidant activity of compounds present in this plant, thus investigating the antioxidant potential of lemongrass through "in vitro" method of 2,2 difenil-1-picril hidrazil radical (DPPH) was the aim of the present work. The free radical sequestering activity of $\mathrm{EBH}$ from lemongrass leaves was
\end{abstract}


significant, demonstrating concentration-dependent effect, which can be attributed to the presence of flavonoids, catechins and other phenolic compounds.

KEYWORDS: Lemongrass, Cymbopogon citratus, Antioxidant Activity, Medicinal Plants and DPPH.

\section{INTRODUÇÃO}

O capim-cidreira (Cymbopogon citratus) é uma espécie herbácea pertencente à família Poaceae, que engloba aproximadamente 500 gêneros e 8.000 espécies (EKPENYONG, 2015; AJAYI, 2016). É uma planta originária do continente asiático, mas amplamente distribuída nas regiões tropicais e subtropicais, adaptável a variadas condições de clima e solo, preferindo climas quentes e úmidos (GUPTA ; JAIN, 1978; AVOSEH, 2015). É uma planta aromática, perene, frondosa e robusta, de rizomas curtos e formam touceiras. Suas folhas são moles, longas, finamente estriadas e cortantes, coloração verde pálida e apresentam odor agradável (NUNES, 2010).

O uso popular da planta se faz na forma de chá das suas folhas, utilizada no Brasil e no mundo, cuja finalidade abrange uma ampla indicação terapêutica. Seu uso comercial baseia-se principalmente em preparações para o chá e ainda a utilização de substâncias extraídas da planta, especialmente do óleo essencial, utilizado na indústria de alimentos e cosméticos (DUBEY et al., 1997). O chá do capim cidreira é indicado popularmente para o stress, dor, febre, tosse e alterações digestivas, sendo que constituintes extraídos do óleo essencial da planta demonstra efeitos antiespasmódico, antibacteriano, analgésico (COSTA et al., 2005; EKPENYONG, 2015; EBANI, 2018), fungicida (ALMEIDA et al 2008; BOUKHATEM, 2014), ansiolítica e sedativa (BLANCO et al 2009).

As folhas e rizomas do capim-cidreira possuem diferentes flavonóides como luteolina, quercetina, campferol, isoorientina e isoscoparina (CHEEL, et al, 2005), sendo descrito também a presença de alcalóides, terpenóides e taninos (AVOSEH, et al., 2015). O óleo essencial extraído das folhas contém maior quantidade substâncias do grupo dos monoterpenos, como o citral $(65-86 \%)$ e cintronelal $(7-8 \%)$ (SACCHETTI et al., 2005), apresentando maior quantidade nas folhas jovens, sendo o composto responsável pelo odor característico da planta (SOUZA et al., 2005), componente que juntamente com os flavonoides são notoriamente conhecidos por suas atividades antioxidantes vinculadas a atividade terapêutica (AVOSEH, et al., 2015), através da atividade de neutralização de radicais exercida por esta classe de componentes, principalmente à sua estrutura química, do número e posição dos grupos hidroxila e da natureza das substituições nos anéis aromáticos com propriedades de oxirredução (HASLAM, 1996; SOARES, 2002). Estas substâncias apresentam-se amplamente distribuídas, sendo encontradas em diferentes plantas do reino vegetal (KARAKAYA, 2004).

Os antioxidantes naturais provenientes do extrato de plantas são preferíveis por sua baixa toxicidade em relação aos antioxidantes sintéticos (WOLFE et al., 2003; PALIPOCH, 2013), sendo ainda associado a uma menor incidência de patologias relacionadas ao estresse oxidativo (DROGE, 2002), área de crescente interesse científico, visto a grande variedade de compostos com propriedades antioxidantes, além da complexidade quanto ao modo de combate aos radicais livres (LU; FOO, 2000; XU, 2017). Com isso, o estudo da atividade antioxidante de plantas medicinais pode dar origem à escolha de possíveis candidatos a estudos farmacológicos vinculados a esta propriedade bioquímica. 
Dentre diferentes metodologias para avaliação da atividade antioxidante de compostos naturais, a atividade sequestradora do radical livre 2,2- difenil-1-picrilhidrazila - DPPH é utilizada como recurso bioquímico por sua ação frente a um determinado antioxidante ou uma espécie radicalar $(R \bullet)$, sendo o DPPH•com coloração originalmente púrpura, reduzido a difenil-picril-hidrazina, que possui coloração amarela, monitorada pelo decréscimo da absorbância em uma banda de 520 nm (MOLYNEUX, 2004; PISOSCHI, 2016).

Com estes recursos, a investigação das propriedades bioquímicas do capimcidreira pode ser realizada e mensurada por sua atuação contra os radicais livres, possibilitando uma defesa antioxidante satisfatória pelos componentes disponíveis para inativá-los. Assim, determinadas patologias que são ocasionadas pelos danos oxidativos induzidos nas células e tecidos, podem ser prevenidos ou revertidos através da possível propriedade antioxidante do composto, consistindo no objeto de investigação deste trabalho através da avaliação da capacidade desta planta em sequestrar radicais livres, ou seja, o potencial antioxidante através do método "in vitro" 2,2 difenil-1-picril hidrazil radical (DPPH), com o intuito de agregar valor a essa planta.

\section{MATERIAL E MÉTODOS}

Mudas de capim-cidreira (Cymbopogon citratus) foram adquiridas e plantadas no Horto de Plantas Medicinais da Universidade Estadual de Goiás - Campus Itumbiara e após atingirem a fase adulta, coletadas, secas à temperatura ambiente por 12 dias, moída, peneirada e armazenada sob refrigeração em recipiente hermeticamente fechado até sua utilização.

Para os procedimentos experimentais, foi obtido o extrato bruto hidroalcoólico $(\mathrm{EBH})$ das folhas do capim-cidreira, processo em que 25 gramas de folha moída e triturada foram adicionadas a $500 \mathrm{~mL}$ de solvente hidroalcoólico $70 \%(350 \mathrm{~mL}$ de etanol $+150 \mathrm{~mL}$ de água destilada), em seguida, realizadas agitações periódicas pelo período de 72 horas. Após esse tempo, a mistura foi filtrada e levada para a estufa à $40^{\circ} \mathrm{C}$ até a completa secagem do solvente. $\mathrm{O}$ rendimento do $\mathrm{EBH}$ foi de 3,6 gramas $(14,4 \%)$, o qual foi armazenado sob refrigeração (entre $2-8^{\circ} \mathrm{C}$ ) até sua utilização.

A atividade antioxidante foi avaliada pelo método "in vitro" 2,2 difenil-1-picril hidrazil radical (DPPH), ensaio que avalia a redução do radical estável DPPH por compostos antioxidantes, promovendo a mudança de coloração púrpura à amarela, proporcional à concentração da substância redutora da amostra, permitindo o cálculo da concentração necessária para reduzir $50 \%$ do radical DPPH $\left(\mathrm{IC}_{50}\right)$. À partir do EBH foram preparadas soluções em tubos com DPPH $(0,3 \mathrm{M})$ nas concentrações de 3, 10, 30, 100, 300 e $1000 \mathrm{~g} / \mathrm{mL}$. Todos os tubos permaneceram incubados por 30 minutos à temperatura ambiente na ausência de luz, em seguida foi realizada a leitura em espectrofotômetro em $515 \mathrm{~nm}$.

A capacidade antioxidante do capim-cidreira em eliminar o radical DPPH foi determinada segundo a equação descrita por KULISC et al (2004):

$$
\% \text { da atividade antioxidante }=\frac{(\text { Abs controle }- \text { Abs amostra })}{\text { Abs controle }}
$$

Abs controle: Absorbância da solução de DPPH sem a amostra.

Abs amostra: Absorbância da amostra em diferentes concentrações com o DPPH. 
À partir dos dados obtidos, plotou-se um gráfico de regressão linear e calculou-se o valor da $\mathrm{IC}_{50}$, ou seja, a concentração da amostra necessária para inibir $50 \%$ do radical DPPH.

\section{RESULTADOS E DISCUSSÃO}

A planta em estudo obteve desenvolvimento satisfatório, atingindo touceiras de aproximadamente um metro de altura, com coloração e aroma agradáveis, características peculiares de sua espécie. $O$ bom desenvolvimento destas plantas permitiu a coleta de folhas saudáveis e bom padrão de qualidade. A qualidade das plantas medicinais depende de vários fatores, incluindo variações de espécies, clima, coleta, armazenamento e processamento, que influenciam na produção de metabólitos quimicamente ativos pela planta (SOUZA et al., 2009; AVOSEH, 2015).

O capim-cidreira adapta-se a condições variadas de clima e solo, preferindo climas quentes e úmidos, com chuvas bem distribuídas e temperatura média elevada (NUNES, 2010), condições essas que permitiram um alto rendimento da planta, visto que no local em que foram cultivadas, prevalece-se um clima tropical, com uma estação de seca que ocorre no outono/inverno e outra chuvosa que ocorre na primavera/verão. A qualidade, quantidade e duração da luz são características determinantes nos rendimentos desta planta, visto sua posição vertical que possibilita melhor utilização da luz, fatores que corroboram com as afirmações de Ortiz et al. (2002) que inclusive relata que sua posição vertical possibilita uma melhor utilização da luz, fator que juntamente com o aumento da temperatura e duração do dia, determina o rápido crescimento da massa verde, alcançando a maturidade em menor tempo.

Dentre diferentes metodologias para a investigação do efeito antioxidante, o método DPPH possibilita a realização do ensaio "in vitro" preciso e efetivo, amplamente utilizado, pois requer um tempo relativamente curto em relação a outros métodos e que possibilita efetiva mensuração do consumo de radicais livres para avaliação do efeito antioxidante de diferentes compostos.

Radicais livres são moléculas orgânicas e inorgânicas ou átomos que contêm um ou mais elétrons não pareados na última camada eletrônica, com existência independente. São altamente reativos, capaz de reagir com qualquer composto situado próximo à sua órbita 12 externa, passando a ter uma função oxidante ou redutora (HALLIWELL, 1994; VALKO, 2007). São subprodutos do metabolismo aeróbio, normalmente, a presença de radicais livres na célula, situa-se em níveis toleráveis, o que possibilita uma defesa antioxidante satisfatória pelos meios disponíveis para inativá-los (KASOTE, 2015), mas sua produção excessiva de radicais livres ou redução nas defesas antioxidantes do organismo, promovem estresse oxidativo nas células (FINKEL ; HOLBROOK et al., 2000). Desta forma, o estudo de compostos que provem de plantas medicinais, mostram-se uma fonte inesgotável de recursos para diferentes finalidades terapêuticas.

Antioxidantes, de forma geral, são qualquer substância que presente em concentrações baixas, quando comparadas ao substrato oxidável, atrasa ou inibe a oxidação do substrato de maneira eficaz (BARREIROS, 2006) e com este estudo foi visto que a atividade sequestradora de radicais livres do EBH das folhas de capimcidreira mostrou-se significante, demonstrando efeito dependente de concentração. A menor concentração utilizada ( $3 \mathrm{~g} / \mathrm{mL}$ ), não apresentou nenhum decréscimo no consumo de DPPH, considerando, portanto, uma atividade antioxidante $0 \%$ nesta concentração, diferente da próxima concentração analisada $(10 \mathrm{~g} / \mathrm{mL})$ que apresentou início de decréscimo no consumo de DPPH. Efeitos mais consideráveis 
foram vistos nas concentrações de 30, 100 e $300 \mathrm{~g} / \mathrm{mL}$, merecendo destaque a concentração de $1000 \mathrm{~g} / \mathrm{mL}$ que demonstrou decréscimo de $100 \%$ no consumo de $\mathrm{DPPH}$. Com este experimento, o capim-cidreira demonstrou possuir atividade antioxidante por meio do sequestro do radical DPPH, permitindo o cálculo da concentração da amostra necessária para inibir $50 \%$ do radical DPPH $\left(\mathrm{IC}_{50}\right)$.

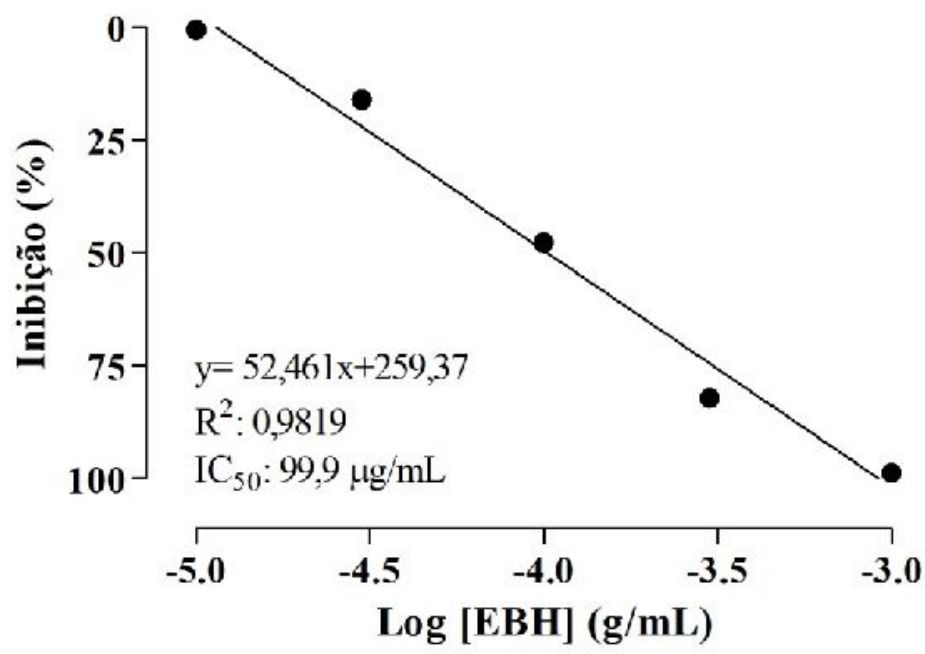

FIGURA 1 Equação de regressão linear da atividade antioxidante do EBH de campim-cidreira (Cymbopogon citratus) através da metodologia "in vitro" do 2,2 difenil-1-picril hidrazil radical (DPPH).

Com a equação de regressão linear da atividade antioxidante do EBH do capim-cidreira, obtido através do cálculo da redução percentual do radical estável $\mathrm{DPPH}$, por compostos antioxidantes obteve a seguinte equação da reta: y = $52,461 x+259,37$. Através desta equação, pode-se encontrar a concentração necessária para inibir $50 \%$ do radical DPPH que correspondeu a $99,9 \mu \mathrm{g} / \mathrm{mL}$. Neste procedimento experimental foi possível mensurar a correlação linear de $R^{2}=0,9819$, representando uma linearidade de aproximadamente $98 \%$.

O solo de cultivo, as condições climáticas, características genéticas da planta, condições de secagem, método de preparação do EBH e tempo de armazenamento, demonstrou-se efetivo e passível de reprodutibilidade, logo, infere-se que a planta em estudo não sofreu interferências ambientais que comprometesse sua atividade bioquímica. Nestas condições, analisando a propriedade bioquímica do EBH do capim-cidreira, entende-se que esta planta possui atividade antioxidante, e que este efeito pode justificar as diversas finalidades terapêuticas em seu uso popular, contribuindo na investigação do potencial terapêutico desta planta.

\section{CONCLUSÕES}

O EBH das folhas do capim-cidreira (Cymbopogon citratus) cultivado no Horto de Plantas Medicinais da UEG - Campus Itumbiara, demonstrou significante atividade sequestradora de radicais livres, com efeito dependente de concentração, comprovando seu efeito antioxidante frente ao DPPH e possibilitando correlações com os benefícios na medicina popular. 


\section{REFERÊNCIAS}

AJAYI, E. O.; SADIMENKO, A. P.; AFOLAYAN, A. J. GC-MS evaluation of Cymbopogon citratus (DC) Stapf oil obtained using modified hydrodistillation and microwave extraction methods. Food Chemistry, v. 15, n. 209, 262-6, 2016. Disponível

em: https://www.sciencedirect.com/science/article/pii/S0308814616305970?via\%3Dihub. https://doi.org/10.1016/j.foodchem.2016.04.071

ALMEIDA, R. B. A.; CARRETTO, C. F. P.; SANTANA, R. S.; FURLAN, M. R.; JUNQUEIRA, J. C.; JORGE, A. O. C. Atividade antimicrobiana de Cymbopogon citratus (DC.) stapf sobre Candida spp. Revista de Odontologia da UNESP, v. 37, n.2, 147-153, 2008. Disponível em: http://s3.amazonaws.com/host-articleassets/rou/588018447f8c9d0a098b4b48/fulltext.pdf.

AVOSEH, O.; OYEDEJI, O.; RUNGQU, P.; NKEH-CHUNGAG, B.; OYEDEJI, A. Cymbopogon Species; Ethnopharmacology, Phytochemistry and the Pharmacological Importance, Molecules, v. 20, n. 5, 7438-53, 2015. Disponível em: https://www.mdpi.com/1420-3049/20/5/7438.

https://doi.org/10.3390/molecules20057438.

BARREIROS, A. L. B. S.; DAVID, J. M.; DAVID, J. P. Estresse oxidativo: relação entre espécies reativas de oxigênio e a defesa do organismo. Química Nova, v. 29, n. $113,2006.2$ Disponível em: http://quimicanova.sbq.org.br/imagebank/pdf/Vol29No1_113_20-DV04221.pdf.

BLANCO, M. M.; COSTA C. A. R. A.; FREIRE A. O.; SANTO J. G. JR.; COSTA M. Neurobehavioral effect of essential oil of Cymbopogon citratus in mice. Phytomedicine: International Journal of Phytotherapy and Phytopharmacology, v. 16, 265-70, 2009. Disponível em: https://ac.els-cdn.com/S0944711307000712/1s2.0-S0944711307000712-main.pdf?_tid=21ee1d36-567e-4191-a0b76b155b3ac9cd\&acdnat=1552502896_7ec97e58b2f529bdeb14bf97d422fe20. doi:10.1016/j.phymed.2007.04.007.

BOUKHATEM, M. N.; FERHAT, M. A.; KAMELI, A.; SAIDI, F.; KEBIR, H. T. Lemon grass (Cymbopogon citratus) essential oil as a potent anti-inflammatory and antifungal drugs, Libyan Journal of Medical Sciences, v. 9, 2014. Disponível em: https://www.tandfonline.com/doi/full/10.3402/ljm.v9.25431.

https://doi.org/10.3402/ljm.v9.25431.

CHEEL, J.; THEODULOZ, C .; RODRIAGUEZ, J .; SCHMEDA-HIRSCHMANN, G. Removedores de radicais livres e antioxidantes de capim-limão (Cymbopogon citratus (DC.) Stapf.). Journal of Agricultural and Food Chemistry, v. 53, 2511-17, 2005. Disponível em: https://ac.els-cdn.com/S0023643895800085/1-s2.0S0023643895800085-main.pdf?_tid=f9fb18e4-da07-465c-af02-

3ab8f33c4b2a\&acdnat=1552503051_3784fcf92ff2d3480b8351452330f67e. https://doi.org/10.1016/S0023-6438(95)80008-5.

COSTA, L. C. B.; CORRÊA, R. M.; CARDOSO, J. C. W.; PINTO, J. E. B. P.; BERTOLUCCI, S. K. V.; FERRI, P. H. Secagem e fragmentação da matéria seca no rendimento e composição do óleo essencial de capim-limão. Horticultura Brasileira, Brasília, v.23, n.4, 956-59, 2005. Disponível em: 
http://www.scielo.br/pdf/hb/v23n4/a19v23n4.

http://dx.doi.org/10.1590/S0102-

05362005000400019.

DROGE W. Free radicals in the physiological control of cell function. Physiological

Reviews, v. 82, n. 1, 47-95, 2002. Disponível em: https://www.physiology.org/doi/full/10.1152/physrev.00018.2001?url_ver=Z39.88-

2003\&rfr_id=ori\%3Arid\%3Acrossref.org\&rfr_dat=cr_pub\%3Dpubmed\&\#. doi: 10.1152 / physrev.00018.2001.

DUBEY, N. K.; NISHORE, N.; VARMA,J.; LEE, S.Y.; Citotoxicity of essential oils of Cymbopogon citratus and Ocinun gratissimum. Indian Journal of Pharmaceutical Sciences, Kalina, v. 59, n. 5, 263-64, 1997. Disponível em: http://www.ijpsonline.com/articles/cytotoxicity-of-the-essential-oils-of-cymbopogoncitratus-and-ocimum-gratissimum.pdf.

EBANI, V. V.; BASMA, N.; BERTELLONI, F.; PISTELLI, L.; MANCIANTI, F.; NARDONI, S. Chemical Composition and In Vitro Antimicrobial Efficacy of Sixteen Essential Oils against Escherichia coli and Aspergillus fumigatus Isolated from Poultry, Journal of Veterinary Science, v. 5, n. 3, 62, 2018. Disponível em: https://www.ncbi.nlm.nih.gov/pmc/articles/PMC6164321/pdf/vetsci-05-00062.pdf. doi: 10.3390/vetsci5030062.

EKPENYONG, C. E.; AKPAN, E.; NYOH, A. Ethnopharmacology, phytochemistry, and biological activities of Cymbopogon citratus (DC.) Stapf extracts. Chinese Journal of Natural Medicines, v. 13, n. 5, 321-37, 2015. Disponível em: https://ac.els-cdn.com/S1875536415300236/1-s2.0-S1875536415300236-

main.pdf? tid=1596068d-0108-4615-864f-

b0ab9c5d21b0\&acdnat=1552503912_91cfbaf3e556ee9216dcf5025cb8ab22. doi: 10.1016 / S1875-5364 (15) 30023-6.

FINKEL, T.; HOLBROOK, N. J. Oxidants, oxidative stress and the biology of ageing. Nature, v. 408, n. 6809, 239-47, 2000. Disponível em: https://doi.org/10.1038/35041687. DOI: 10.1038 / 35041687.

GUPTA, B. K.; JAIN, N. Cultivation and utilization of Genus Cymbopogon in Indian. Indian Perfumer, New Delhi, v. 22, n. 2 , 55-68, 1978.

HALLIWELL, B. Free radicals and antioxidants: a personal view. Nutrition Reviews, New York, v. 52, n. 8, 253-65, 1994. Disponível em: https://www.ncbi.nlm.nih.gov/pubmed/7970288.

HASLAM, E.; Natural Polyphenols (Vegetable Tannins) as Drugs: Possible Modes of Action. Journal of Natural Products, v. 59, n. 205, 1996. Disponível em: https://pubs.acs.org/doi/10.1021/np960040\%2B. doi:10.1021/np960040+.

KARAKAYA, S. Bioavailability of Phenolic Compounds. Critical Reviews in Food Science and Nutrition, Boca Raton, v. 44, n. 6, 453-64, 2004. Disponível em: https://www.tandfonline.com/doi/abs/10.1080/10408690490886683.

https://doi.org/10.1080/10408690490886683. 
KASOTE, D. M.; KATYARE, S. S.; HEGDE, M. V.; BAE, H. Significance of Antioxidant Potential of Plants and its Relevance to Therapeutic Applications. International Journal of Biological Sciences, v. 11, n. 8, 982-91, 2015. Disponível em: https://www.ncbi.nlm.nih.gov/pmc/articles/PMC4495415/. doi: 10.7150 / ijbs.12096.

KULISIC, T.; RADONIC, A.; KATALINIC, V.; MILOS, M. Use of different methods for testing antioxidative activity of oregano essential oil. Food Chemistry, Barking, v. 85, n. 4, 633-40, 2004. Disponível em: https://www.sciencedirect.com/science/article/pii/S0308814603003741. https://doi.org/10.1016/j.foodchem.2003.07.024.

LU, Y.; FOO, L. Y. Antioxidant and radical scavengig activities of polyphenols from apple pomace. Food Chemistry, v. 68, n. 25, 81-85, 2000. Disponível em: https://bmcchem.biomedcentral.com/articles/10.1186/s13065-016-0195-7.

https://doi.org/10.1186/s13065-016-0195-7.

MOLYNEUX, P. The use of the stable free radical diphenylpicrylhydrazyl (DPPH) for estimating antioxidant activity. Songklanakarin Journal of Science and Technology, v. 26, n. 2, 211-19, 2004. Disponível em: http://www.thaiscience.info/journals/Article/SONG/10462423.pdf.

NUNES, S. E. A. Subsídios para implantação do programa de fitoterapia no município de Imperatriz, estado do Maranhão. Dissertação de Mestrado em Gestão, Pesquisa e Desenvolvimento em Tecnologia Farmacêutica - Pontifícia Universidade Católica de Goiás, 2010. Disponível em: http://tede2.pucgoias.edu.br:8080/bitstream/tede/2135/1/Sheila\%20Elke\%20Araujo\% 20Nunes.pdf.

ORTIZ, R. S.; MARRERO, G. V.; NAVARRO, A. L. T. Instructivo técnico del cultivo de Cymbopogon citratus (DC.) Stapf (caña santa). Revista Cubana de Plantas Medicinales, v. 7, n. 2, 89-95, 2002. Disponível em: http://scielo.sld.cu/scielo.php?script=sci_arttext\&pid=S1028-47962002000200007.

PALIPOCH S. A review of oxidative stress in acute kidney injury: protective role of medicinal plants-derived antioxidants. African journal of traditional, complementary, and alternative medicines, African Journal of Traditional, Complementary and Alternative Medicines, v.10, n.4, 88-93, 2013. Disponível em: https://www.ncbi.nlm.nih.gov/pmc/articles/PMC3794397/.

PISOSCHI, A. M.; POP, A.; CIMPEANU, C.; PREDOI, G. Antioxidant Capacity Determination in Plants and Plant-Derived Products: A Review. Oxidative Medicine And Cellular Longevity, 2016. Disponível em: https://www.hindawi.com/journals/omcl/2016/9130976/. http://dx.doi.org/10.1155/2016/9130976.

SACCHETTI, G.; MAIETTI, S.; MUZZOLI, M.; SCAGLIANTI, M.; MANFREDINI, S.; RADICE, M.; BRUNI, R. Comparative evaluation of 11 essential oils of different origin as functional antioxidants, antiradicals and antimicrobials in foods. Food Chemistry, v. 91, n. $4,621-32,42005.4$ Disponível em: 
https://www.sciencedirect.com/science/article/pii/S0308814604005096.

https://doi.org/10.1016/j.foodchem.2004.06.031.

SOARES, S. E. Ácidos fenólicos como antioxidantes. Revista de Nutrição, v. 15, n. 1, 71-81, 2002. Disponível em: http://www.scielo.br/pdf/rn/v15n1/a08v15n1.pdf.

SOUZA, E. L.; LIMA, E. O.; FREIRE, K. R. L.; SOUSA, C. P. Inhibitory action of some essential oils and phytochemicals on the growth of various molds isolated from foods. Brazilian Archives of Biology and Technology, v. 48, n. 2, p. 245-250, 2005. UNIVERSITY of Hawaii. Botany Department. Poaceae (Gramineae). Disponível em: http://www.scielo.br/pdf/babt/v48n2/23764.pdf. http://dx.doi.org/10.1590/S1516-89132005000200011

VALKO, M.; LEIBFRITZ, D.; MONCOL, J.; CRONIN, M. T.; MAZUR, M.; TELSER, J. Free radicals and antioxidants in normal physiological functions and human disease. The International Journal of Biochemistry \& Cell Biology, v. 39, 44-84, 2007. Disponível em: https://www.sciencedirect.com/science/article/pii/S1357272506002196?via\%3Dihub. doi: 10.1016 / j.biocel.2006.07.001.

WOLFE, K.; WU, X.; LIU, R. H. Antioxidant activity of apple peels. Journal of Agricultural and Food Chemistry, v. 53, 609-14, 2003. Disponível em: https://pubs.acs.org/doi/pdf/10.1021/jf020782a. DOI: 10.1021 / jf020782a.

XU, D. P.; LI, Y.; MENG, X.; ZHOU, T.; ZHOU, Y.; ZHENG, J.; ZHANG, J. J.; Li, H. B. Natural Antioxidants in Foods and Medicinal Plants: Extraction, Assessment and Resources. International journal of molecular sciences, v. 18, n. 1, 96, 2017. Disponível em: https:/www.mdpi.com/1422-0067/18/1/96. https://doi.org/10.3390/ijms18010096. 